\title{
A Cluster Head Decision System for Sensor Networks Using Fuzzy Logic and Number of Neighbor Nodes
}

\author{
Junpei Anno ${ }^{\dagger}$, Leonard Barolli ${ }^{\ddagger}$, Arjan Durresi ${ }^{\dagger \dagger}$, Fatos Xhafa ${ }^{\dagger \ddagger}$, Akio Koyama ${ }^{\star \ddagger}$ \\ ${ }^{\dagger}$ Graduate School of Engineering \\ Fukuoka Institute of Technology (FIT) \\ 3-30-1 Wajiro-Higashi, Higashi-Ku, Fukuoka 811-0295, Japan \\ E-mail:mgm07001@bene.fit.ac.jp \\ ${ }^{\ddagger}$ Department of Information and Communication Engineering \\ Fukuoka Institute of Technology (FIT) \\ 3-30-1 Wajiro-Higashi, Higashi-Ku, Fukuoka 811-0295, Japan \\ E-mail: barolli@fit.ac.jp \\ ${ }^{\dagger \dagger}$ Department of Computer and Information Science \\ Indiana University Purdue University Indianapolis \\ 723 W. Michigan Street SL 280, Indianapolis, IN 46202, USA \\ E-mail:durresi@cs.iupui.edu \\ ${ }^{*}$ Department of Languages and Informatics Systems \\ Polytechnic University of Catalonia \\ Jordi Girona 1-3, 08034 Barcelona, Spain \\ E-mail:fatos@lsi.upc.edu \\ *Department of Informatics, Yamagata University \\ 4-3-16 Jonan, Yonezawa 992-8510, Yamagata, Japan \\ E-mail:akoyama@eie.yz.yamagata-u.ac.jp
}

\begin{abstract}
Cluster formation and cluster head selection are important problems in sensor network applications and can drastically affect the network's communication energy dissipation. However, the selection of cluster head is not easy in different environments which may have different characteristics. In this paper, in order to deal with this problem we propose a power reduction algorithm for sensor networks based on fuzzy logic and number of neighbor nodes. We evaluate the proposed system by simulations and show that proposed system makes a good selection of the cluster head.
\end{abstract}

\section{Introduction}

Recent developments in technologies such as wireless communication and microelectronics have enabled Wireless Sensor Network (WSN) applications to be deployed for many applications such as battlefield surveillance and environment monitoring. An important aspect of such networks is that the nodes are unattended, resource-constrained, their energy cannot be replenished and network topology is unknown. The resource-constrained limitations make it essential for these sensor nodes to conserve energy to increase life-time of the sensor network [1, 2, 3, 4].

Recently, there are a lot of research efforts towards the optimization of standard communication paradigms for such networks. In fact, the traditional Wireless Network (WN) design has never paid attention to constraints such as the limited or scarce energy of nodes and their computational power. Also, in WSN paths can change over time, because of time-varying characteristics of links, local contention level and nodes reliability. These problems are important especially in multi-hop scenarios [4].

There are many fundamental problems that sensor networks research will have to address in order to ensure a reasonable degree of cost and system quality. Some of these problems include sensor node clustering, Cluster Head $(\mathrm{CH})$ selection and energy dissipation. There are many research works that deal with these challenges $[5,6,7,8,9,10,11,12,13]$.

The cluster based algorithms could be used for 
partitioning the sensor nodes into subgroups for task subdivision or energy management. Cluster formation is one of most important problems in sensor network applications and can drastically affect the network's communication energy dissipation. Clustering is performed by assigning each sensor node to a specific $\mathrm{CH}$. All communication to (from) each sensor node is carried out through its corresponding $\mathrm{CH}$ node. Obviously one would like to have each sensor to communicate with the closest $\mathrm{CH}$ node to conserve its energy, however $\mathrm{CH}$ nodes can usually handle a specific number of communication channels. Therefore, there is a maximum number of sensors that each $\mathrm{CH}$ node can handle. This does not allow each sensor to communicate to its closest $\mathrm{CH}$ node, because the $\mathrm{CH}$ node might have already reached its service capacity. $\mathrm{CHs}$ can fuse data from sensors to minimize the amount of data to be sent to the sink. When network size increases, clusters can also be organized hierarchically.

In the conventional cluster architecture, clusters are formed statically at the time of network deployment. The attributes of each cluster, such as the size of a cluster, the area it covers, and the members it possesses, are static. In spite of its simplicity, the static cluster architecture suffers from several drawbacks. The fixed membership is not robust from the perspective of fault tolerance. If a $\mathrm{CH}$ dies of power depletion, all the sensors in the cluster render useless. Also, fixed membership prevents sensor nodes in different clusters from sharing information and collaborating on data processing. Dynamic cluster architectures, on the other hand, offer several desirable features. Formation of a cluster is triggered by certain events of interest.

In this work we deal with the $\mathrm{CH}$ selection. When a sensor with sufficient battery and computational power detects (with a high Signal-to-Noise Ratio: SNR) signals of interest, it volunteers to act as a $\mathrm{CH}$. This is a simple method, because no explicit leader $(\mathrm{CH})$ election is required and, hence, no excessive message exchanges are incurred. However, the selection of $\mathrm{CH}$ in this way is not easy in different environments which may have different characteristics.

The heuristic approaches based on Fuzzy Logic (FL) and Genetic Algorithms (GA) can prove to be efficient for traffic control in wireless networks $[14,15]$.

In our previous work [16], we proposed a fuzzybased cluster selection method for wireless sensor networks, which uses 3 parameters for $\mathrm{CH}$ selection: Distance of Cluster Centroid, Remaining Battery Power of Sensor and Network Traffic. The performance of our method was better than a previous method [17]. But, we found that for $\mathrm{CH}$ selection the number of neighbor nodes is very important. For this reason, in this paper we propose and implement a new $\mathrm{CH}$ system using FL and number of neighbor nodes.

The paper is organized as follows. In Section 2, we discuss the related work. In Section 3, we introduce the proposed system design. In Section 4, we present the simulation results. Conclusions are given in Section 5.

\section{Related Work}

In this section, we review related work in clustering algorithms. Several clustering methods such as weighted clustering [5], hierarchal clustering [6] and dynamic clustering [7] algorithms have been proposed to organize nodes as a cluster. Most algorithms elect leaders based on certain weights or iteratively optimize a cost function or use heuristic to generate minimum number of clusters. The Distributed Clustering Algorithm (DCA) [8] assumes quasi-stationary nodes with real-valued weights. The Weighted Clustering Algorithm [5] elects a node based on the number of neighbors, transmission power and so on. The MaxMind-Clustering Algorithm [9] generates d-hop clusters with a run time of $\mathrm{O}(\mathrm{d})$ rounds. This algorithm does not minimize the communicating complexity of sending information to the information center.

The hierarchal clustering scheme [6] uses spanning tree-based approach to produce cluster with certain properties. However, energy efficiency is not addressed in this work. In [10], the authors have proposed an emergent algorithm that iteratively tries to achieve high packing efficiency, however negotiation among nodes to be $\mathrm{CH}$ and join cluster based on degree and proximity leads to high amount of communication overhead, thus wastage energy.

LEACH [11, 12] uses two-layered architecture for data dissemination. In this scheme, sensors periodically elect themselves as $\mathrm{CHs}$ with some probability and broadcast an invitation message for nearby nodes to join the cluster. The nodes that do not 
intend to be $\mathrm{CHs}$ join the cluster based on the proximity of $\mathrm{CH}$, thus minimizing the communicating cost. However, LEACH and PEGASIS [13] require the apriory knowledge of the network topology.

In [17], the authors propose a self-reconfiguring protocol for Wireless Personal Area Networks (WPAN) using an unsupervised clustering method.



Figure 1. FLC structure.


Figure 2. Triangular and trapezoidal membership functions.

Their fuzzy logic system is used to select the master/controller for each cluster. In our previous work [16], we had shown by simulation results that the selection surface of our system was better than the system in [17].

\section{Proposed System Model}

We propose and implement a new $\mathrm{CH}$ system based on FL and number of neighbor nodes.

The Fuzzy Logic Controller (FLC) basic elements are shown in Fig. 1. They are the fuzzifier, inference engine, Fuzzy Rule Base (FRB) and defuzzifier.

As shown in Fig. 2, as membership functions we use triangular and trapezoidal membership functions because they are suitable for real-time operation [18].

In Fig. $2, x_{0}$ in $f(x)$ is the center of triangular function; $x_{0}\left(x_{1}\right)$ in $g(x)$ is the left (right) edge of trapezoidal function; and $a_{0}\left(a_{1}\right)$ is the left (right) width of the triangular or trapezoidal function.

In our previous system as the input parameters for FLC we used: Distance of Cluster Centroid, Remaining Battery Power of Sensor and Network Traffic. However, we found that the number of the neighbor nodes is very important for the selection of the $\mathrm{CH}$. To explain this effect let us consider a small network model with 14 nodes as shown in Fig. 3. In this figure, the node number 1 has 6 neighbor nodes, for this reason is selected as a $\mathrm{CH}$. After that, 2 other sets of nodes remain, but node 2 has more neighbor nodes than nodes 3 and 7 , thus node 2 is selected as $\mathrm{CH}$.

By considering the number of neighbor nodes as a fuzzy input parameter, we propose 3 input linguistic parameters for our system:

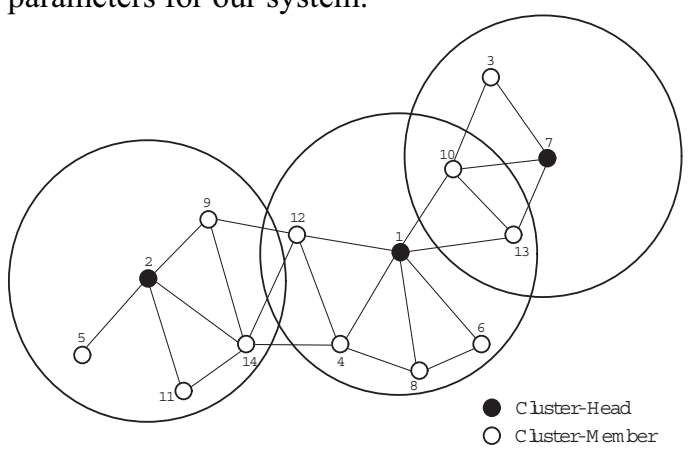

Figure 3. CH selection process.

- Remaining Battery Power of Sensor (RPS);

- Degree of Number of Neighbor Nodes (D3N);

- Distance from Cluster Centroid (DCC).

The term sets for each input linguistic parameter are defined respectively as:

$T(R P S)=\{\operatorname{Low}($ Lo $)$, Middle $(\mathrm{Mi}), \operatorname{High}(\mathrm{Hg})\} ;$

$T(D 3 N)=\{F e w(F w)$, Medium $(M e)$, Many $(M n)\}$;

$T(D C C)=\{$ Near $(\mathrm{Nr})$, Moderate $(\mathrm{Mo}), \mathrm{Far}(\mathrm{Fr})\}$.

The membership functions for input parameters of FLC are defined as:

$$
\begin{aligned}
& \mu_{L o}(R P S)=g\left(R P S ; L o_{0}, L o_{1}, L o w_{0}, L o_{w 1}\right) ; \\
& \mu_{M i}(R P S)=f\left(R P S ; M i_{0}, M i_{w 0}, M i_{w 1}\right) ; \\
& \mu_{H g}(R P S)=g\left(R P S ; H g_{0}, H g_{1}, H g_{w 0}, H g_{w 1}\right) ; \\
& \mu_{F w}(D 3 N)=g\left(D 3 N ; F w_{0}, F w_{1}, F w_{w 0}, F w_{w 1}\right) ; \\
& \mu_{M e}(D 3 N)=f\left(D 3 N ; M e_{0}, M e_{w 0}, M e_{w 1}\right) ; \\
& \mu_{M n}(D 3 N)=g\left(D 3 N ; M n_{0}, M n_{1}, M n_{w 0}, M n_{w 1}\right) ; \\
& \mu_{N r}(D C C)=g\left(D C C ; N r_{0}, N r_{1}, N r_{w 0}, N r_{w 1}\right) ; \\
& \mu_{M o}(D C C)=f\left(D C C ; M o_{0}, M o_{w 0}, M o_{w 1}\right) ; \\
& \mu_{F r}(D C C)=g\left(D C C ; F r_{0}, F r_{1}, F r_{w 0}, F r_{w 1}\right) .
\end{aligned}
$$

The small letters $w 0$ and $w 1$ mean left width and right width, respectively.

The output linguistic parameter is the Possibility of $\mathrm{CH}$ Selection $(\mathrm{PCHS})$. We define the term set of PCHS as:

$\{$ Very Weak $(V W)$, Weak $(W)$, Little Weak $(L W)$,

Medium (MD), Little Strong (LS), Strong (S), Very

Strong $(V S)\}$.

The membership functions for the output parameter $P C H S$ are defined as:

$\mu_{V W}(P C H S)=g\left(P C H S ; V W_{0}, V W_{1}, V W w_{0}, V W_{w 1}\right) ;$ 
$\mu_{W}(P C H S)=f\left(P C H S ; W_{0}, W_{w 0}, W_{w 1}\right) ;$

$\mu_{L W}(P C H S)=f\left(P C H S ; L W_{0}, L W_{w 0}, L W_{w 1}\right) ;$

$\mu_{M D}(P C H S)=f\left(P C H S ; M D_{0}, M D_{w 0}, M D_{w 1}\right) ;$

$\mu_{L S}(P C H S)=f\left(P C H S ; L S_{0}, L S_{w 0}, L S_{w 1}\right)$;

$\mu_{S}(P C H S)=f\left(P C H S ; S_{0}, S_{w 0}, S_{w 1}\right)$;

$\mu_{V S}(P C H S)=g\left(P C H S ; V S_{0}, V S_{1}, V S_{w 0}, V S_{w 1}\right)$.

Table 1. Parameters and their term sets.

\begin{tabular}{|c|c|}
\hline Parameters & Term Sets \\
\hline Remaining Battery & Low, Middle, High \\
Power of Sensor (RPS) & \\
Degree of Number of & Few, Medium, Many \\
Neighbor Nodes (D3N) & \\
Distance from Cluster & Light, Moderate, Heavy \\
Centroid (DCC) & Very Weak, \\
\hline Possibility of & Weak, Little Weak, \\
& Medium, Little Strong, \\
CH Selection (PCHS) & Strong, Very Strong \\
\hline
\end{tabular}
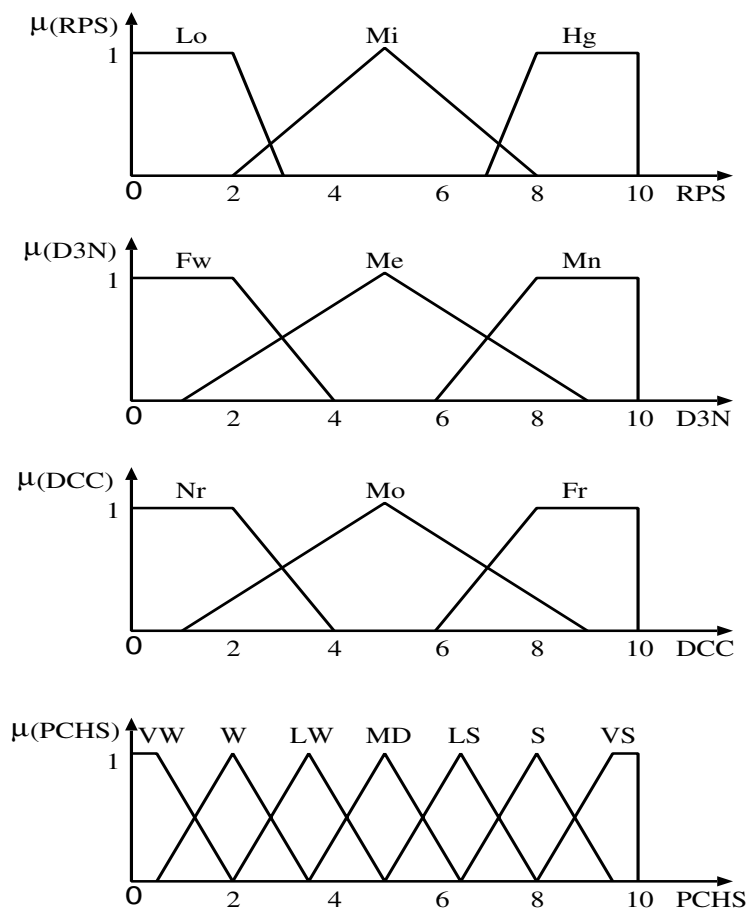

Figure 4. Membership functions.

The linguistic parameters and their term sets of proposed system are shown in Table 1. The fuzzy membership functions for input parameters are shown in Fig. 4.
The FRB is shown in Table 2 and forms a fuzzy set of dimensions $|T(R P S)| \times|T(D 3 N)| \times|T(D C C)|$, where $|T(x)|$ is the number of terms on $T(x)$. The FRB has 27 rules. The control rules have the form: IF "conditions" THEN "control action".

Table 2. FRB.

\begin{tabular}{|c|c|c|c|c|}
\hline Rule & RPS & D3N & DCC & PCHS \\
\hline 1 & Lo & Fw & Fr & VW \\
2 & Lo & Fw & Mo & W \\
3 & Lo & Fw & Nr & W \\
4 & Lo & Me & Fr & W \\
5 & Lo & Me & Mo & W \\
6 & Lo & Me & Nr & W \\
7 & Lo & Mn & Fr & VW \\
8 & Lo & Mn & Mo & VW \\
9 & Lo & Mn & Nr & VW \\
10 & $\mathrm{Mi}$ & $\mathrm{Fw}$ & $\mathrm{Fr}$ & W \\
11 & $\mathrm{Mi}$ & $\mathrm{Fw}$ & $\mathrm{Mo}$ & LW \\
12 & $\mathrm{Mi}$ & $\mathrm{Fw}$ & $\mathrm{Nr}$ & $\mathrm{MD}$ \\
13 & $\mathrm{Mi}$ & $\mathrm{Me}$ & $\mathrm{Fr}$ & $\mathrm{LW}$ \\
14 & $\mathrm{Mi}$ & $\mathrm{Me}$ & $\mathrm{Mo}$ & $\mathrm{MD}$ \\
15 & $\mathrm{Mi}$ & $\mathrm{Me}$ & $\mathrm{Nr}$ & $\mathrm{LS}$ \\
16 & $\mathrm{Mi}$ & $\mathrm{Mn}$ & $\mathrm{Fr}$ & $\mathrm{MD}$ \\
17 & $\mathrm{Mi}$ & $\mathrm{Mn}$ & $\mathrm{Mo}$ & $\mathrm{LS}$ \\
18 & $\mathrm{Mi}$ & $\mathrm{Mn}$ & $\mathrm{Nr}$ & $\mathrm{S}$ \\
19 & $\mathrm{Hg}$ & $\mathrm{Fw}$ & $\mathrm{Fr}$ & $\mathrm{LW}$ \\
20 & $\mathrm{Hg}$ & $\mathrm{Fw}$ & $\mathrm{Mo}$ & $\mathrm{MD}$ \\
21 & $\mathrm{Hg}$ & $\mathrm{Fw}$ & $\mathrm{Nr}$ & $\mathrm{LS}$ \\
22 & $\mathrm{Hg}$ & $\mathrm{Me}$ & $\mathrm{Fr}$ & $\mathrm{MD}$ \\
23 & $\mathrm{Hg}$ & $\mathrm{Me}$ & $\mathrm{Mo}$ & $\mathrm{LS}$ \\
24 & $\mathrm{Hg}$ & $\mathrm{Me}$ & $\mathrm{Nr}$ & $\mathrm{S}$ \\
25 & $\mathrm{Hg}$ & $\mathrm{Mn}$ & $\mathrm{Fr}$ & $\mathrm{LS}$ \\
26 & $\mathrm{Hg}$ & $\mathrm{Mn}$ & $\mathrm{Mo}$ & $\mathrm{S}$ \\
27 & $\mathrm{Hg}$ & $\mathrm{Mn}$ & $\mathrm{Nr}$ & $\mathrm{VS}$ \\
\hline
\end{tabular}

\section{Simulation Results}

In this section, we present the simulation results. We evaluate by computer simulations the performance of the previous systems $[16,17]$ and the proposed system. In our system, we decided the number of term sets by carrying out many simulations.

In Fig. 5 is shown the performance evaluation of the previous system [17]. The graph in 3 dimensions shows the relation between the probability of a sensor to be selected as a $\mathrm{CH}$ versus the distance and the remained sensor power. With the increase of the 
remained sensor power and the decrease of the distance between the sensor and sink, the probability of a sensor to be selected as $\mathrm{CH}$ is increased. However, the graph surface is increased almost proportionally with the changes of both parameters.

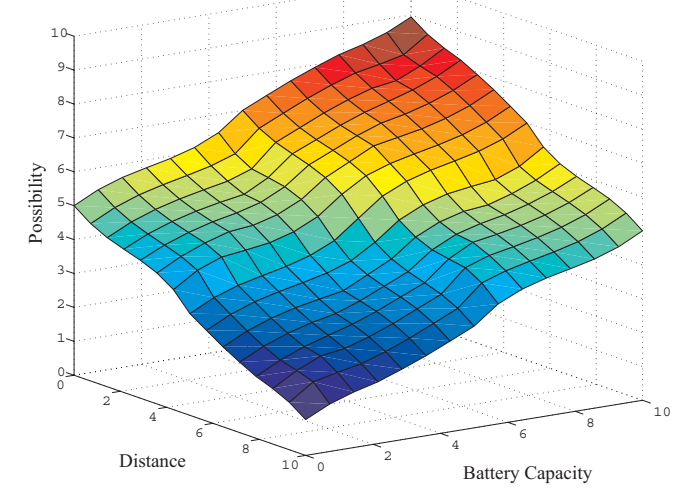

Figure 5. Previous system simulation results.

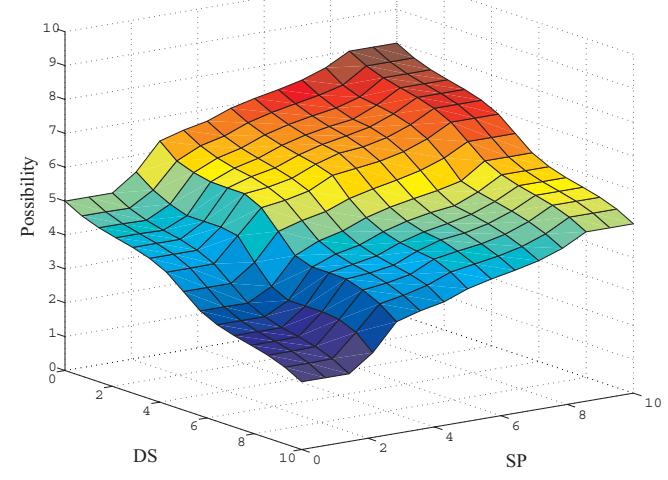

Figure 6. Previous fuzzy system results.

The performance evaluation of our previous implemented system is shown in Fig. 6 [16]. This is the same relation with the previous system. With the increase of the remained sensor power and the decrease of the distance between the sensor and the sink, the possibility of a sensor to be selected as $\mathrm{CH}$ is increased. The surface control of the fuzzy-based system is better than previous system.

We show the performance of the proposed system in Figs. 7, 8 and 9. In Fig. 7 is shown the same relation with previous systems. The $\mathrm{CH}$ selection surface of proposed system is better than the other systems. We clearly distinguish 3 zones. When, the RPS is less than 2 units the probability of a node to be selected as $\mathrm{CH}$ is very small (first zone). A middle zone (more than 2 units but less than 8 units): where the $\mathrm{CH}$ selection possibility increases slowly with increase of RPS and decrease of DCC. A third zone (more than 8 units): where the possibility of a node to be $\mathrm{CH}$ is high. Thus, the proposed system can control better the RPS, which results in the increase of the network lifetime.

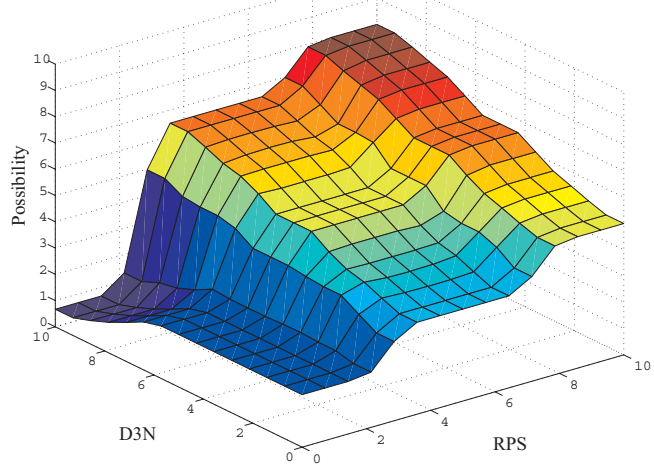

Figure 7. Proposed system results (case 1).

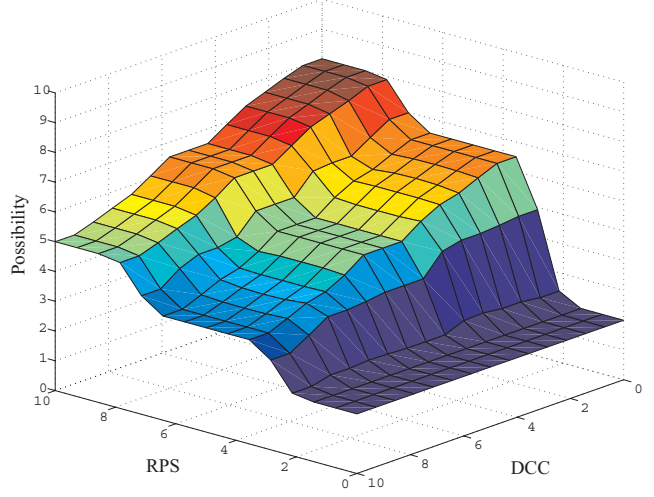

Figure 8. Proposed system results (case 2).

In Fig. 8 is shown the relation between the possibility of a sensor to be selected as a $\mathrm{CH}$ versus the D3N and RPS. Also, here we see 3 zones. When the RPS is low and the number of neighbor nodes (D3N) is high, the possibility that a node is selected as $\mathrm{CH}$ is low. When the RPS is middle and the D3N increases, the possibility that a node is selected as $\mathrm{CH}$ is increased. When the RPS is high and the D3N is high, the possibility that a node is selected as $\mathrm{CH}$ is high.

In Fig. 9, we show the relation between possibility of a sensor to be selected as a $\mathrm{CH}$ versus the $\mathrm{D} 3 \mathrm{~N}$ and DCC. With the increase of $\mathrm{D} 3 \mathrm{~N}$ and the decrease of DCC, the possibility of a sensor to be selected as $\mathrm{CH}$ is 
increased. Different from the results in Fig. 7 and 8, we see that the shape of the surface is changed proportionally with the changes of parameters. This shows that the RPS and $3 \mathrm{DN}$ parameters are more important for the selection of a $\mathrm{CH}$ than the DCC.

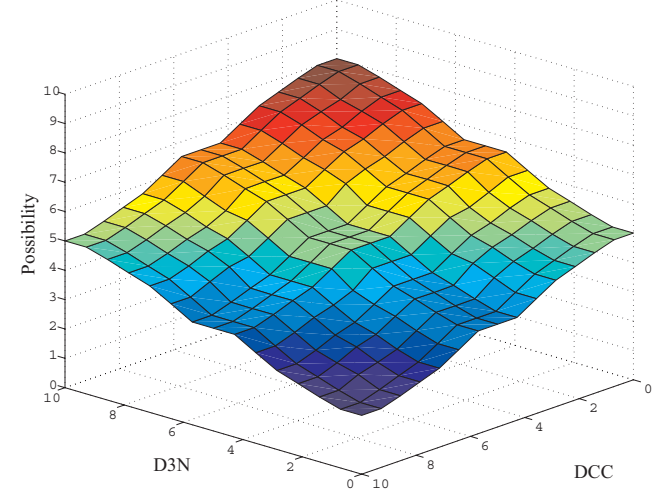

Figure 9. Proposed system results (case 3).

\section{Conclusions}

The power conservation in WSN is a very important issue. Clustering is one of the energy-efficient techniques for extending the lifetime of a sensor network. However, $\mathrm{CH}$ selection is very difficult when many parameters are used for making the decision.

In this paper, we proposed a $\mathrm{CH}$ selection system based on FL and the number of neighbor nodes. We evaluated the proposed system by computer simulations. The proposed system shows better performance than 2 previous systems. The remained energy of the sensor and the number of neighbor nodes are more important parameters than the distance of the node from the sink.

We are working now to implement the proposed system in NS-2 simulator in order to compare the performance with other non fuzzy-based approaches such as LEACH.

\section{Acknowledgment}

The authors would like to thank the Japanese Society for the Promotion of Science (JSPS) for the financial support.

\section{References}

[1] I. F. Akyildiz, W. Su, Y. Sankarasubramaniam, and E. Cayirci, "Wireless Sensor Networks: A Survey", Computer Networks, Vol. 38, No. 4, pp. 393-442, 2002.
[2] I. F. Akyildiz and I. H. Kasimoglu, "Wireless Sensor and Actor Networks: Research Challenges", Ad Hoc Networks, Vol. 2, No. 4, pp. 351-367, 2004.

[3] S. Giordano and C. Rosenberg, "Topics in AdHoc and Sensor Networks", IEEE Communication Magazine, Vol. 44, No. 4, pp. 97-97, 2006.

[4] J. N. Al-Karaki and A. E. Kamal, "Routing Techniques in Wireless Sensor Networks: A Survey”, IEEE Wireless Communication, Vol. 11, No. 6, pp. 6-28, December 2004.

[5] M. Chatterjee, S. K. Das, and D. Turgut, "WCA: A Weighted Clustering Algorithm for Mobile Ad Hoc networks", Journal of Cluster Computing, Vol. 5, No. 2, pp. 193-204, April 2002.

[6] S. Banerjee and S. Khuller, "A Clustering Scheme for Hierarchical Control in Multi-hop Wireless Networks", Proc. of IEEE INFOCOM-2001, pp. 1028-1037, 2001.

[7] W. P. Chen, J. C. How, and L. Sha, "Dynamic Clustering for Acoustic Target Tracking in Wireless Sensor Networks", IEEE Trans. on Mobile Computing, Vol. 3, No. 3, pp. 258-271, July-September 2004.

[8] S. Basagni, "Distributed Clustering for Ad Hoc Networks", International Symposium of Parallel Architectures, Algorithms and Networks (I-SPAN'99), pp. 310-315, 1999.

[9] A. D. Amis, R. Prakash, T. H. P. Vuong, and D. T. Huynh, "Max-min D-cluster Formation in Wireless Ad Hoc Networks", Proc. of IEEE INFOCOM-2000, pp. 3241, 2000.

[10] H. Chan and A. Perrig, "ACE: An Emergent Algorithm for Highly Uniform Cluster Formation”, Proc. of European Workshop on Wireless Sensor Networks (EWSN-2004), pp. 154-171, 2004.

[11] W. B. Heinzelman, A. P. Chandrakasan, and H. Balakrishnan, "An Application-specific Protocol Architecture for Wireless Microsensor Networks", IEEE Transactions on Wireless Communications, Vol. 1, No. 4, pp. 660-670, October 2004.

[12] W. R. Heinzelman, A. Chandrakasan, and H. Balakrishnan, "Energy-efficient Communication Protocol for Wireless Microsensor Networks", Proc. of the 33rd Annual Hawaii International Conference on System Sciences (HICSS-2000), pp. 3005-3014, 2000.

[13] S. Lindsey, C. Raghavendra, and K. M. Sivalingam, "Data Gathering Algorithms in Sensor Networks Using Energy Metrics", IEEE Trans. on Parallel and Distributed Systems, Vol. 13, No. 9, pp. 924-935, 2002.

[14] P. M. L. Chan, R. E. Sheriff, Y. Hu, P. Conforto, and C. 
Tocci, "Mobility Management Incorporating Fuzzy Logic for a Heterogeneous IP Environment", IEEE Communications Magazine, Vol. 39, No. 12, pp. 42-51, 2001.

[15] L. Barolli, A. Koyama, T. Suganuma, and N. Shiratori, "Gaman: A GA Based QoS Routing Method for Mobile Ad-hoc Networks", Journal of Interconnection Networks (JOIN), Vol. 4, No. 3, pp. 251-270, 2003.

[16] J. Anno, L. Barolli, F. Xhafa, and A. Durresi, "A Cluster Head Selection Method for Wireless Sensor Networks Based on Fuzzy logic", Proc. of IEEE TENCON-2007, CD-ROM, 4 pages, 2007.

[17] Q. Liang, "A Design Methodology for Wireless Personal Area Networks with Power Efficiency", Proc. of the Wireless Communications and Networking (WCNC), Vol. 3, pp. 1475-1480, 2003.

[18] J. M. Mendel, "Fuzzy Logic Systems for Engineering: A Tutorial", Proc. of the IEEE, Vol. 83, No. 3, pp. 345377, 1995. 